\title{
Academic Appointments and Vacancies
}

\section{APPOINTMENTS}

AdAMs, Theodore S., Instr. Eng., Univ. of Buffalo, Buffalo, N.Y.

Batrd, James R., Asst. Prof. Eng., Connecticut Coll., New London, Conn.

Betsky, Seymodr, Asst. Prof. Eng., Wellesley Coll., Wellesley, Mass.

Brown, Donald F., Prof. and Head Dept. Spanish, Hope Coll., Holland, Mich.

Carrier, Warren P., Asst. Prof. Eng., Univ. of Iowa, Iowa City, Iowa.

Carter, H. Everett, Asst. Prof. Eng., Univ. of California, Berkeley 4, Calif.

Dickinson, Jaures R., Instr. Eng., Univ. of Nevada, Reno, Nev.

EDDY, FREDERICK D., Prof. and Head Dept. Modern and Classical Langs., Hood Coll., Frederick, Md.

Farrere, Helene F., LaGrange Coll., LaGrange, Ga.

Gaubert, Helen A., Eng. Dept., State Teachers Coll., Plattsburgh, N.Y.

Hinrichs, JohanN J., Stamford Coll., Stamford, Conn.

HiRsch, HaNs E., State Teachers Coll., Plattsburgh, N.Y.

Huld, Vernam, Prof. Celtic Lang. and Lit., Harvard Univ., Cambridge 38, Mass.

Ketcham, Carl H., Instr. Eng., Univ. of Arizona, Tucson, Ariz.

KIlburn, Patrick E., Instr. Eng., Univ. of Colorado, Boulder, Colo.

KNELLER, JoHN W., Instr. French, Oberlin Coll., Oberlin, Ohío.

Laberee, John Alfred, President, Ricker Coll., Houlton, Me.

Lawn, Woodrow, H., Prof. Humanities, Webb Instit, of Naval Architecture, Glen Cove, N.Y.

DE Mandach, ANDRE, Dept. Modern Langs., Princeton Univ., Princeton, N.J.

Portuondo, Jose Antonio, Asst. Prof. Spanish, Columbia Univ., New York 27, N.Y.
Ringler, Widltam A., JR., Prof. Eng., Washington Univ., St. Louis 5, Mo.

Shockley, Martin S., Prof. Eng., North Texas State Coll., Denton, Tex.

Wadsworth, Phillp A., Assoc. Prof. Romance Langs., Northwestern Univ., Evanston, Ill.

Wannhouse, Austryn, Research Asst. Eng., Univ. of Iowa, Iowa City, Iowa.

Williliams, Arthur R., Asst. Prof. Eng. Univ, of Massachusetts, Amherst, Mass.

Witmarth, Wirson E., Colorado A. \& M. Coll., Fort Collins, Colo.

ZitTad, Herbert G., Instr. French, Lawrence Coll., Appleton, Wis.

\section{VACANCIES*}

Untv, of Arkansas, Fayetteville, Ark.; English; Ph.D.; instr., lit. and comp.; $\$ 3000-3400$; address A. Howard Carter, chro.

BEREA Coll., Berea, Kentucky; instr.; French; 1950-51 only; address Louis Smith, Dean.

DuQuesne Univ., Pittsburgh 19, Pa.; speech, play directing; salary not below $\$ 3000$; address J. M. Purcell, chm.

Duquesne Univ., Pittsburgh 19, Pa.; English; American lit. Ph.D.; salary not below $\$ 3000$; address J. M. Purcell, chm.

Odachita Coll., Arkadelphia, Ark.; English; Ph.D.; head of dept.; minimum salary $\$ 4000$; address J. R. Grant, Pres.

Pennsylvania State Coll., State College, Pa.; Spanish (primarily) \& French; male, Ph.D.; instr.; $\$ 3600$; address R. J. Clements, head, Dept. of Rom. Langs.

Univ. of Redlands, Redlands, Calif; Spanish; Ph.D.; foreign residence; Asst. Prof.; $\$ 3600-\$ 3900$; address George H. Armacost, Pres.

Dalmouste Univ., Halifax, Nova Scotia; prof. of mod. langs. \& head of dept.; address Dean George E. Wilson.

* Letters of application should contain full data on academic experience and publications, and also directions for obtaining credentials. Since many departments lack adequate clerical assistance, applicants should expect no more than a brief, formal acknowledgment of their letters. A reply-postcard might well be enclosed for this purpose. 